\title{
Pre-travel Health Care Utilization Among Travelers Who Visit Friends and Relatives
}

\author{
Eugene M. Tan ${ }^{1 *}$, Jane W. Njeru², Debra J. Jacobson ${ }^{3}$, Patrick M. Wilson ${ }^{3}$, Chun Fan ${ }^{3}$, Jasmine R. Marcelin ${ }^{1}$, \\ Donna J. Springer', Mark L. Wieland ${ }^{2}$, Irene G. Sia ${ }^{1}$ \\ ${ }^{1}$ Division of Infectious Diseases, Mayo Clinic, 200 First St SW, Rochester, MN 55905, USA \\ ${ }^{2}$ Division of Primary Care Internal Medicine, Mayo Clinic, 200 First St SW, Rochester, MN 55905, USA \\ ${ }^{3}$ Division of Biomedical Statistics and Informatics, Mayo Clinic, 200 First St SW, Rochester, MN 55905, USA
}

Corresponding Author: Eugene M. Tan, M.D., Division of Infectious Diseases, Mayo Clinic, 200 First St SW, Rochester, MN 55905, Phone: 1-507-293-7781, Fax: 1-507-255-7767, E-mail: tan.eugene@mayo.edu

Received April 26, 2017; Accepted June 21, 2017; Online Published June 30, 2017

\begin{abstract}
Introduction: Immigrants to the United States who return home to visit friends and relatives (VFRs) have high rates of travel-related infections. The data on VFR utilization of pre-travel health care is inadequate. The objective of this study was to describe the travel patterns and adherence to pre-travel recommendations of VFRs.

Methods: This retrospective study compared pre-travel health care utilization between VFR and non-VFR patients in one travel clinic from 2012-2013. Study investigators reviewed patients' electronic medical records for demographic data, travel characteristics, and rates of immunizations and preventive medication prescriptions (i.e. antimalarial prophylaxis and antibiotics for traveler's diarrhea). Categorical variables were compared using chi-square tests. Multivariate logistic regression was used to model adjusted associations of VFR with completion of pre-travel recommendations.

Results: VFRs $(n=393)$ were younger than non-VFRs $(n=1680)$, more often required interpreters for language translation, and more commonly had government insurance coverage than non-VFRs. VFRs were more likely to travel to lower-income countries in Africa, Asia, and the Middle East. VFRs had longer durations of travel: 51\% for $>4$ weeks vs. $21 \%$ for non-VFRs $(P<0.0001)$. VFRs were less likely to complete tetanus, polio, and rabies vaccinations, but more likely to complete measles, mumps, and rubella vaccinations. Only the association with the rabies vaccination remained significant after adjustment $(\mathrm{OR}[95 \% \mathrm{Cl}]=0.3$ $[0.1,0.8])$.

Conclusion: VFRs had longer travel durations and lower rates of vaccine completion than non-VFRs. More research is needed to understand this disparity and to promote changes in practice.

Keywords: Emigrants and immigrants, Vaccination, Immunization, Travel medicine
\end{abstract}

Citation: Tan EM, Njeru JW, Jacobson DJ, et al. Pre-travel health care utilization among travelers who visit friends and relatives. Int J Travel Med Glob Health. 2017;5(2):53-59. doi:10.15171/ijtmgh.2017.11.

\section{Introduction}

In the past 30 years, many immigrants to higher-income regions of North America have originated from lower-income countries in Asia, Latin America, the Caribbean, and subSaharan Africa. According to the United Nations, 244 million international migrants, including 20 million refugees, were living abroad in 2015. ${ }^{1}$ In the United States in 2013, $13 \%$ of the population (40 million) was foreign-born. ${ }^{2}$ These immigrants and their children often return to their countries of origin to visit friends and relatives (VFRs). ${ }^{3}$ One study reported that $23 \%$ (2.1 million) of 9.1 million international travelers were VFRs. ${ }^{4}$ Minnesota is experiencing rapid growth of its foreignborn population; from 1990-2015, the number of Asian and African residents tripled, and the number of Hispanic residents quadrupled. ${ }^{5}$ According to the Minnesota State Demographic Center, the nonwhite population is estimated to grow from $14 \%$ in 2005 to $25 \%$ in $2035 .{ }^{6}$ Therefore, the rate of international VFR travel is likely to increase.

Compared with tourist travelers, VFRs have higher travelassociated health risks. ${ }^{7,8}$ VFRs have a higher incidence of travel-related infections such as typhoid fever, tuberculosis, and hepatitis $\mathrm{A}$, and they are 8 times more likely to receive a diagnosis of malaria. ${ }^{3}$ In a 2011 study of 772 Quebecois travelers, VFRs accounted for $53 \%$ of malaria cases, $57 \%$ of hepatitis A cases, and $94 \%$ of typhoid cases. ${ }^{9}$ The Centers for Disease Control and Prevention (CDC) list several reasons underlying the increased prevalence of travel-related illnesses in VFRs, including lack of awareness of risk, low rate of

Copyright (C) The Author(s). This is an open access article distributed under the terms of the Creative Commons Attribution License (http:// creativecommons.org/licenses/by/4.0), which permits unrestricted use, distribution, and reproduction in any medium, provided the original work is properly cited. 
pre-travel health care utilization, and financial and cultural barriers. ${ }^{3}$ While abroad, VFRs tend to stay with family members and may quickly re-adopt local lifestyle practicesfor example, they may live in crowded conditions, sleep without window screens, and consume untreated water and uncooked food. ${ }^{7}$

Given these high travel-related health risks, VFRs may benefit from pre-travel consultation. However, only approximately $31.4 \%$ of VFRs (compared with $60.9 \%$ of tourist travelers) seek pre-travel advice. ${ }^{8}$ Furthermore, information on pre-travel assessment of VFRs is limited. This study aimed to characterize VFRs and non-VFRs who sought pre-travel consultation at a local travel clinic and describe the VFRs' travel patterns and adherence to pre-travel recommendations. For those VFRs who sought pre-travel health care, this study compared adherence with recommended vaccines between VFRs and non-VFRs. This study aimed to assess these measures, as past studies on VFRs suggested this population may be at higher risk of travel-related infection due to longer durations of stay and lower adherence with pre-travel medical advice. $^{10}$

\section{Methods}

Pre-travel Health Care

A retrospective cohort study of all patients (VFRs and nonVFRs) who received pre-travel health care at the Mayo Clinic Travel and Tropical Medicine Clinic (TTMC) from January 1, 2012, through December 31, 2013 was conducted. The Mayo TTMC is the largest travel clinic serving residents of Olmsted County, Minnesota. Consultations there include an assessment of the following four primary topics: vaccines, malaria, diarrhea, and safety. Based on the patient's travel itinerary and medical and immunization history, the travel medicine provider assesses the risk for vaccine-preventable illness, mosquito-transmitted illnesses (particularly malaria), traveler's diarrhea, and environmental and safety hazards. Counseling is provided regarding risks and benefits of recommended routines and travel-related vaccines, rabies post-exposure prevention after animal bites, mosquito avoidance measures, malaria chemoprophylaxis, food and water precautions, and self-management of traveler's diarrhea with an antidiarrheal and antibiotic. Recommendations for vaccines and preventive travel-related medications (e.g., malaria chemoprophylaxis and an antibiotic for self-treatment of traveler's diarrhea) are provided in accordance with CDC guidelines. ${ }^{11}$

\section{Data Collection and Measures}

All children and adults who sought pre-travel consultation at the Mayo TTMC from January 1, 2012 through December 31, 2013 met the inclusion criteria for this study. The pretravel consultation at the Mayo TTMC is standardized, and all patients seeking pre-travel consultation are provided a questionnaire that asks about the purpose of travel. Visiting friends and relatives is listed as one of the reasons for travel on the questionnaire. Those who selected "Visiting Friends and Relatives" were included in the VFR cohort. VFRs were defined as immigrants and refugees who returned to their countries of origin to visit their friends and relatives. ${ }^{3}$ Patients were excluded if they did not have a Minnesota Research
Authorization, which authorized the review of medical records for study purposes.

The following data was abstracted from the electronic health records of all TTMC patients: demographics (age, gender, race/ethnicity, VFR status, and insurance type); travel characteristics (time from travel clinic visit to date of departure, destination, and duration of travel); immunizations; and anti-malarial and antibiotic prescriptions. Place of travel was categorized into 5 groups: (1) Africa, (2) Asia,( 3) Latin America, Europe, the Caribbean, or Australia, (4) Middle East, and (5) missing data or unknown. These categories were not mutually exclusive, because travelers could list multiple countries. Dates were obtained by reviewing pre-travel consultation clinical notes, which are based on the patient's responses to the standardized questionnaire provided prior to the appointment. During the pre-travel consultation, the health care provider elicits and documents any additional travel-related information as needed.

Recommendations for and completion of travel-related vaccines (hepatitis A, hepatitis B, Japanese encephalitis, rabies, typhoid, and yellow fever) and routine vaccines (human papillomavirus, influenza, measles-mumps-rubella [MMR], meningococcus, pneumococcus, polio, tetanus-diphtheriapertussis, and zoster) were recorded in the immunization module within the electronic medical record as administered, declined, or deferred (with rationale for deferral). Vaccine recommendations were based on the patient's travel itinerary and medical and immunization history, following current CDC guidelines. ${ }^{11}$

At the Mayo TTMC, serologic testing is often performed when travelers report a potential history of vaccination or infection for hepatitis A, hepatitis B, MMR, or varicella, especially if the traveler is deemed to be at high risk of acquiring any of these infections during travel. If serologic testing is negative (i.e., no serologic evidence of immunity), then patients are advised to undergo vaccination; if serologic testing is positive (i.e., serologic evidence of immunity is present), patients are considered to have completed the specific vaccination recommendations.

Vaccine completion was defined as receipt (with documentation) or confirmed positive serology of the recommended vaccine (influenza, MMR, meningococcus, pneumococcus, polio, typhoid, varicella, zoster, yellow fever) or completion of the entire multi-dose series (hepatitis A, hepatitis $\mathrm{B}$, human papillomavirus, Japanese encephalitis, and rabies). If the vaccine series was only partially completed, it was categorized as a partial or incomplete vaccination. For children, polio vaccine completion was defined as completion of the series; for adults, polio vaccination was considered complete with receipt of one booster injection, when indicated. If a patient refused a recommended vaccine or did not have adequate time to complete a recommended vaccine series, it was considered an incomplete vaccine. Only vaccines that were recommended on the basis of the traveler's specific itinerary or risks (as determined by the health care provider) were included in the analysis of completed vaccines.

Preventive medication prescriptions included malaria chemoprophylaxis (atovaquone-proguanil, chloroquine, doxycycline, and mefloquine) and antibiotics (azithromycin, ciprofloxacin, and levofloxacin) for presumptive treatment of 
traveler's diarrhea.

\section{Data Analysis and Modeling}

All data analyzed during this study is included in this published article. Demographic and travel data were categorized and summarized as frequencies and percentages. Completed vaccinations were summarized by number and percentage. The $\chi^{2}$ test was used to assess differences in demographics, travel characteristics, vaccinations, and antimalarial and antibiotic prescriptions between VFRs and non-VFRs. Multivariable analysis with logistic regression was used to adjust for factors that were significant with univariate analysis. Results of the logistic regression models are presented as odds ratios (OR) and $95 \%$ confidence intervals (CIs). Statistical significance was defined as $P \leq 0.05$. All analyses were performed using SAS version 9.4 (SAS Institute Inc.).

\section{Results}

In total, 393 (19.0\%) VFRs and 1,680 (81.0\%) non-VFRs who were seen in the TTMC during the study period were identified (Table 1). Among VFRs, slightly more were female $(n=206$ [52.4\%]), but for non-VFRs, the numbers of females $(n=855[50.9 \%])$ and males $(n=825[49.1 \%])$ were similar. Significantly more VFRs than non-VFRs were in the age range of $0-20$ years $(101$ [25.7\%] vs 185 [11.0\%]; $P<0.001)$. Only 54 (13.7\%) VFRs were white, whereas 1,487 (88.5\%) of non-VFRs were white $(P<0.001)$. A greater proportion of VFRs required interpreters during their clinic visits compared with nonVFRs (75 [19.1\%] vs 8 [0.5\%]; $P<0.001)$. More VFRs were covered by government insurance (e.g., Medicaid) compared with non-VFRs (109 [27.7\%] vs 18 [1.1\%]; $P<0.001)$.

Whereas most non-VFRs traveled to Latin America, Europe, the Caribbean, or Australia (10.7\% VFR vs 51.6\% non-VFR), more VFRs traveled to Africa (46.8\% VFR vs $29.5 \%$ non-VFR), Asia (39.2\% VFR vs $30.1 \%$ non-VFR), and the Middle East (10.7\% VFR vs $6.1 \%$ non-VFR) $(P \leq 0.001$ for all). VFRs planned longer durations of travel: 202 (51.4\%) VFRs planned to travel for greater than four weeks compared with $345(20.5 \%)$ non-VFRs $(P<0.001)$. More VFRs than non-VFRs were seen within one week of their travel date (92/390 [23.6\%] vs 144/1,676 [8.6\%]; $P<0.001)$.

Compared with non-VFRs, VFRs had significantly lower rates of completion $(P<0.05$ for all $)$ for numerous vaccinations (presented as VFR vs non-VFR, respectively): tetanus ( $80.8 \%$ vs $91.1 \%$ ); polio ( $86.1 \%$ vs $92.9 \%)$; rabies ( $12.3 \%$ vs $27.3 \%)$; and zoster (26.3\% vs $69.1 \%)$ (Table 2$)$. VFRs had a higher rate of completion for MMR vaccination (87.1\% vs $67.8 \% ; P=0.01)$. Rates of vaccine completion were low for both groups for hepatitis A, hepatitis B, Japanese encephalitis, and rabies $(40.6 \%, 30.0 \%, 26.3 \%$, and $12.3 \%$ vs $45.0 \%, 33.1 \%, 36.1 \%$, and $27.3 \%$, for VFR and non-VFR, respectively). In contrast, completion rates for typhoid fever and yellow fever vaccinations were similarly high in both groups $(92.6 \%$ and $84.3 \%$ vs $91.1 \%$ and $76.7 \%$, for VFR and non-VFR, respectively).

VFRs were less likely to complete the recommended tetanus, polio, and rabies vaccinations (Table 3 ). Although the difference was not significant for the MMR vaccine, VFRs were more likely to complete it when recommended. After adjusting for age, sex, insurance type, and duration of
Table 1. Traveler Characteristics ( $N=2073)$

\begin{tabular}{|c|c|c|c|}
\hline Characteristic & $\begin{array}{c}\text { VFR }^{\mathrm{a}} \\
(\mathrm{n}=393)\end{array}$ & $\begin{array}{c}\text { Non-VFR } \\
(n=1680) \\
\end{array}$ & $P$ Value \\
\hline Age, y, No. (\%) & & & $<0.001$ \\
\hline $0-20$ & $101(25.7)$ & $185(11.0)$ & \\
\hline$>20-40$ & $113(28.8)$ & $454(27.0)$ & \\
\hline$>40-60$ & $111(28.2)$ & $555(33.0)$ & \\
\hline$>60$ & $68(17.3)$ & $486(28.9)$ & \\
\hline Male sex, No. (\%) & $187(47.6)$ & $825(49.1)$ & 0.59 \\
\hline Race/ethnicity, No. (\%) & & & $<0.001$ \\
\hline White & $54(13.7)$ & $1487(88.5)$ & \\
\hline Black & $136(34.6)$ & $33(2.0)$ & \\
\hline Asian & $113(28.8)$ & $62(3.7)$ & \\
\hline Hispanic & $8(2.0)$ & $32(1.9)$ & \\
\hline Other/unknown & $82(20.9)$ & $66(3.9)$ & \\
\hline Used an interpreter, No. (\%) & $75(19.1)$ & $8(0.5)$ & $<0.001$ \\
\hline Insurance, No. (\%) & & & $<0.001$ \\
\hline Private & $238(60.6)$ & $1325(78.9)$ & \\
\hline Medicare & $33(8.4)$ & $276(16.4)$ & \\
\hline Medicaid & $109(27.7)$ & $18(1.1)$ & \\
\hline Other & $13(3.3)$ & $61(3.6)$ & \\
\hline Duration of travel, wk, No. (\%) & & & $<0.001$ \\
\hline$\leq 1$ & $8(2.0)$ & $292(17.4)$ & \\
\hline$>1-4$ & $183(46.6)$ & $1043(62.1)$ & \\
\hline$>4-12$ & $116(29.5)$ & $126(7.5)$ & \\
\hline$>12$ & $67(17.0)$ & $92(5.5)$ & \\
\hline Unknown & $19(4.8)$ & $127(7.6)$ & \\
\hline $\begin{array}{l}\text { Time from travel clinic visit to } \\
\text { date of departure, wk, No. }(\%)^{b}\end{array}$ & & & $<0.001$ \\
\hline$\leq 1$ & $92(23.6)$ & $144(8.6)$ & \\
\hline$>1-4$ & $190(48.7)$ & $533(31.8)$ & \\
\hline$>4-12$ & $76(19.5)$ & $630(37.6)$ & \\
\hline$>12$ & $18(4.6)$ & $237(14.1)$ & \\
\hline Unknown & $14(3.6)$ & $132(7.9)$ & \\
\hline \multicolumn{4}{|l|}{ Place of travel, ${ }^{c}$ No. (\%) } \\
\hline Africa & $184(46.8)$ & $495(29.5)$ & $<0.001$ \\
\hline Asia & $154(39.2)$ & $505(30.1)$ & $<0.001$ \\
\hline $\begin{array}{l}\text { Latin America, Europe, Caribbean, } \\
\text { Australia }\end{array}$ & $42(10.7)$ & $866(51.5)$ & $<0.001$ \\
\hline Middle East & $42(10.7)$ & $103(6.1)$ & 0.001 \\
\hline Missing data or unknown & $2(0.5)$ & $5(0.3)$ & 0.52 \\
\hline
\end{tabular}

Abbreviation: VFR, visit friends and relatives.

a VFRs were defined as immigrants and refugees who were returning to their countries of origin to visit friends and relatives.

bercentages were calculated using the following denominators: VFR, $\mathrm{n}$ = 390; non-VFR, $\mathrm{n}=1676$.

c Places of travel were not mutually exclusive. $\chi^{2}$ comparisons of the proportion visiting and not visiting friends and relatives were compared for each place of travel.

travel, VFRs remained less likely to complete tetanus and polio vaccines and more likely to complete MMR vaccines, but these associations were not statistically significant. However, VFRs remained significantly less likely to complete the rabies vaccinations (OR, 0.31 [95\% CI, 0.13-0.77]), even after adjusting for age, sex, insurance type, and duration of travel. Travelers departing more than four weeks after their pre-travel clinic visit had higher odds of completing the rabies vaccine series than those seen fewer than four weeks before 
Table 2. Comparison of Pre-travel Vaccination Rates Among VFR and Non-VFR Travelers

\begin{tabular}{|c|c|c|c|c|c|}
\hline \multirow[b]{2}{*}{ Vaccine } & \multicolumn{2}{|c|}{ VFR $(n=393)$} & \multicolumn{2}{|c|}{ Non-VFR $(n=1680)$} & \multirow[b]{2}{*}{$P$ Value } \\
\hline & $\begin{array}{c}\text { Vaccine } \\
\text { Recommended, No. }\end{array}$ & $\begin{array}{l}\text { Received Vaccine or Had } \\
\text { Positive Serology, No. }(\%)^{\mathrm{a}}\end{array}$ & $\begin{array}{c}\text { Vaccine } \\
\text { Recommended, No. }\end{array}$ & $\begin{array}{l}\text { Received Vaccine or Had } \\
\text { Positive Serology, No. }(\%)^{\mathrm{a}}\end{array}$ & \\
\hline \multicolumn{6}{|c|}{ Routine Vaccines } \\
\hline Tetanus $^{b}$ & 78 & $63(80.8)$ & 406 & $370(91.1)$ & 0.006 \\
\hline Polio $^{c}$ & 101 & $87(86.1)$ & 283 & $263(92.9)$ & 0.04 \\
\hline HPV & 5 & $4(80.0)$ & 40 & $20(50.0)$ & 0.20 \\
\hline Influenza & 107 & $83(77.6)$ & 428 & $323(75.5)$ & 0.65 \\
\hline MMR & 62 & $54(87.1)$ & 59 & $40(67.8)$ & 0.01 \\
\hline Meningococcus & 63 & $59(93.7)$ & 124 & $108(87.1)$ & 0.17 \\
\hline Pneumococcus & 15 & $12(80.0)$ & 77 & $63(81.8)$ & 0.87 \\
\hline Varicella & 31 & $22(71.0)$ & 18 & $15(83.3)$ & 0.33 \\
\hline Zoster & 19 & $5(26.3)$ & 110 & $76(69.1)$ & $<0.001$ \\
\hline \multicolumn{6}{|c|}{ Travel Vaccines } \\
\hline Hepatitis A & 143 & $58(40.6)$ & 814 & $366(45.0)$ & 0.33 \\
\hline Hepatitis B & 40 & $12(30.0)$ & 251 & $83(33.1)$ & 0.70 \\
\hline Japanese encephalitis & 19 & $5(26.3)$ & 61 & $22(36.1)$ & 0.43 \\
\hline Rabies & 81 & $10(12.3)$ & 242 & $66(27.3)$ & 0.006 \\
\hline Typhoidd $^{d}$ & 311 & $288(92.6)$ & 1,192 & $1,086(91.1)$ & 0.40 \\
\hline Yellow fever & 89 & $75(84.3)$ & 326 & $250(76.7)$ & 0.12 \\
\hline No vaccine recommended & 21 & $21(100.0)$ & 114 & $112(98.2)$ & 0.54 \\
\hline
\end{tabular}

Abbreviations: HPV, human papillomavirus; MMR, measles-mumps-rubella; VFR, visiting friends and relatives.

anly those who were recommended to have each vaccine were included in the comparisons. All patients who completed a vaccine series or had positive serology testing were included.

${ }^{\text {b} T e t a n u s ~ v a c c i n e s ~ i n c l u d e d ~ t h e ~ f o l l o w i n g: ~ 1) ~ D T P ~(d i p h t h e r i a, ~ t e t a n u s, ~ a n d ~ p e r t u s s i s) ; ~ 2) ~ T d ~(t e t a n u s ~ a n d ~ d i p h t h e r i a) ; ~ a n d ~ 3) ~ T d a p ~(t e t a n u s, ~ d i p h t h e r i a, ~ a n d ~}$ pertussis).

'Polio vaccine refers to the injectable polio virus vaccine.

'Typhoid vaccine includes both oral and injectable forms.

departure (OR, 2.61 [95\% CI, 1.38-4.93]).

The most common reasons stated for incomplete vaccinations included a perceived low risk of illness $(n=10)$, insufficient time before departure $(n=23)$, fear of adverse effects $(n=5)$, uncertainty regarding vaccination history $(n=3)$, and preference to defer vaccination $(n=4)$. Given the low numbers of documented reasons, no statistical comparisons between VFRs and non-VFRs could be made.

A high number of VFRs (363 [92.4\%]) and non-VFRs (1,560 [92.9\%]) were given antimalarial prescriptions. Significantly more antidiarrheal prescriptions were given to VFRs (233 [59.3\%]) compared with non-VFRs (841 [50.1\%]) $(P<0.001)$.

\section{Discussion}

This study identified several important demographic, travel, and vaccination differences between VFR and non-VFR travelers. Compared with non-VFRs, more VFRs at the travel clinic were young, nonwhite, used interpreters, traveled to Africa and Asia, and traveled for longer durations. Both traveler groups had low vaccine completion rates for hepatitis A, hepatitis B, Japanese encephalitis, and rabies. VFRs were significantly less likely to complete rabies vaccinations than non-VFRs.

The findings highlight several features of VFRs that should prompt changes in pre-travel counseling. Because the study included many younger travelers (age 0-20 years), pediatric patients may be an important subgroup that merits more intensive pre-travel counseling. Although this study did not examine incidence of travel-associated infection in pediatric travelers, others have documented a high rate of traveler's diarrhea in the pediatric population. In a 1991 study of 446 young Swiss travelers, ${ }^{12}$ traveler's diarrhea occurred in 90 of 250 travelers (36.0\%) aged 15 to 20 years. Therefore, children and their parents may require more intensive pre-travel counseling to ensure proper rehydration during bouts of traveler's diarrhea.

In addition, VFRs tend to have longer periods of travel. In the current study, 183 (46.6\%) VFRs planned to travel for at least four weeks. Long-term travel has been associated with increased risk of chronic diarrhea, giardiasis, malaria, schistosomiasis, and leishmaniasis. ${ }^{13}$ VFRs also sought pretravel consultation closer to their departure date than nonVFRs, possibly because of last-minute travel plans for family emergencies. This short timeframe can limit the feasibility of completing vaccine series. ${ }^{11}$ Given these last-minute, longterm travel patterns, primary care providers may want to take a moment during a general medical examination to remind VFRs to seek pre-travel counseling early in the course of travel planning.

Vaccination rates for several preventable illnesses (hepatitis A, hepatitis B, Japanese encephalitis, and rabies) were low for both groups in the current study. These low rates are particularly worrisome for VFRs who often travel to and stay longer in regions in which they have a higher risk of acquiring these illnesses. For example, $61.1 \%$ of viral hepatitis cases among VFRs returning from sub-Saharan Africa were caused by hepatitis B. ${ }^{14}$ VFRs also tend to have closer contact with local residents. In a study of African immigrants living in London, $40.0 \%$ of men and $21.0 \%$ of women had a new sexual partner upon returning to Africa, where the prevalence 
Table 3. Associations Between Patient Characteristics and Completion of Specific Recommended Vaccinations

\begin{tabular}{|c|c|c|c|c|}
\hline \multirow{2}{*}{ Characteristic } & \multicolumn{4}{|c|}{ Odds Ratio $(95 \% \mathrm{CI})$} \\
\hline & Tetanus & MMR & Polio & Rabies \\
\hline \multicolumn{5}{|c|}{ Unadjusted } \\
\hline \multicolumn{5}{|l|}{ VFR } \\
\hline No & Ref & Ref & Ref & Ref \\
\hline Yes & $0.41(0.21-0.79)$ & $2.30(0.97-5.41)$ & $0.47(0.23-0.98)$ & $0.36(0.18-0.74)$ \\
\hline \multicolumn{5}{|l|}{ Age, $y$} \\
\hline $0-20$ & Ref & Ref & Ref & Ref \\
\hline$>20-40$ & $1.09(0.33-3.58)$ & $0.21(0.07-0.63)$ & $0.42(0.05-3.34)$ & $0.77(0.37-1.60)$ \\
\hline$>40-60$ & $1.50(0.46-4.89)$ & $0.93(0.31-2.83)$ & $0.28(0.04-2.25)$ & $0.53(0.24-1.17)$ \\
\hline$>60$ & $1.17(0.37-3.71)$ & $0.68(0.12-3.91)$ & $0.43(0.05-3.56)$ & $0.89(0.39-2.05)$ \\
\hline \multicolumn{5}{|l|}{ Sex } \\
\hline Male & Ref & Ref & Ref & Ref \\
\hline Female & $0.94(0.53-1.69)$ & $2.39(1.04-5.53)$ & $1.12(0.55-2.26)$ & $1.02(0.61-1.69)$ \\
\hline \multicolumn{5}{|l|}{ Race/ethnicity } \\
\hline White & Ref & Ref & Ref & Ref \\
\hline Nonwhite & $0.57(0.31-1.06)$ & $2.92(1.26-6.76)$ & $0.29(0.14-0.61)$ & $0.60(0.34-1.05)$ \\
\hline \multicolumn{5}{|l|}{ Interpreter } \\
\hline No & Ref & Ref & Ref & Ref \\
\hline Yes & $0.44(0.17-1.12)$ & $0.97(0.26-3.69)$ & $0.30(0.13-0.73)$ & $0.38(0.08-1.67)$ \\
\hline \multicolumn{5}{|l|}{ Insurance } \\
\hline Private & Ref & Ref & Ref & Ref \\
\hline Medicaid or Medicare & $0.69(0.38-1.24)$ & $2.10(0.68-6.49)$ & $1.00(0.46-2.17)$ & $0.87(0.46-1.62)$ \\
\hline \multicolumn{5}{|l|}{ Duration of travel, wk } \\
\hline$\leq 4$ & Ref & Ref & Ref & Ref \\
\hline$>4$ & $0.48(0.24-0.94)$ & $2.04(0.83-5.02)$ & $0.59(0.27-1.29)$ & $3.31(1.95-5.62)$ \\
\hline \multicolumn{5}{|c|}{ Adjusted $^{\mathrm{a}}$} \\
\hline \multicolumn{5}{|l|}{ VFR } \\
\hline No & Ref & Ref & Ref & Ref \\
\hline Yes & $0.50(0.23-1.10)$ & $2.76(0.87-8.74)$ & $0.40(0.16-1.00)$ & $0.31(0.13-0.77)$ \\
\hline \multicolumn{5}{|l|}{ Age, y } \\
\hline $0-20$ & Ref & Ref & Ref & Ref \\
\hline$>20-40$ & $1.09(0.32-3.69)$ & $0.38(0.11-1.31)$ & $0.38(0.05-3.09)$ & $0.86(0.36-2.05)$ \\
\hline$>40-60$ & $1.75(0.49-6.25)$ & $3.17(0.79-12.78)$ & $0.36(0.04-3.02)$ & $0.85(0.33-2.20)$ \\
\hline$>60$ & $1.08(0.30-3.86)$ & $0.54(0.08-3.49)$ & $0.30(0.03-2.67)$ & $1.13(0.40-3.21)$ \\
\hline \multicolumn{5}{|l|}{ Sex } \\
\hline Male & Ref & Ref & Ref & Ref \\
\hline Female & $0.95(0.50-1.80)$ & $2.45(0.89-6.73)$ & $0.95(0.44-2.04)$ & $0.95(0.53-1.70)$ \\
\hline \multicolumn{5}{|l|}{ Insurance } \\
\hline Private & Ref & Ref & Ref & Ref \\
\hline Government or other & $0.92(0.43-1.97)$ & $0.97(0.26-3.64)$ & $1.49(0.54-4.10)$ & $0.88(0.38-2.01)$ \\
\hline \multicolumn{5}{|l|}{ Duration of travel, wk } \\
\hline$\leq 4$ & Ref & Ref & Ref & Ref \\
\hline$>4$ & $0.59(0.28-1.21)$ & $1.94(0.68-5.51)$ & $0.73(0.30-1.80)$ & $5.00(2.69-9.28)$ \\
\hline \multicolumn{5}{|c|}{$\begin{array}{l}\text { Time from travel clinic visit to date } \\
\text { of departure, } w k^{b}\end{array}$} \\
\hline$\leq 4$ & - & - & - & Ref \\
\hline$>4$ & - & - & - & $2.61(1.38-4.93)$ \\
\hline
\end{tabular}

Abbreviations: MMR, measles, mumps, rubella; Ref, reference; VFR, visiting friends and relatives.

a Adjusted for VFR, age, sex, insurance, and duration of travel

${ }^{b}$ For rabies, the odds ratio was adjusted for VFR, age, sex, insurance, duration of travel, and time from travel clinic visit to date of departure.

of human immunodeficiency virus and hepatitis B is higher. ${ }^{15}$ The low rate of rabies vaccination (12.3\%) among VFRs in this study has a considerable number of potential implications on travelers' health, because rabies has a near-100\% fatality rate for patients bitten by a rabid animal. ${ }^{16}$

Vaccination series may not be completed for several reasons. A multi-dose vaccine may require up to three clinic visits; therefore, travelers may not have sufficient time before departure. Among VFRs, 92/390 (23.6\%) were seen less than 1 week before departure, and 190/390 (48.7\%) were seen between 1 and 4 weeks before departure. The costs associated with multiple clinic visits also may serve as a disincentive for vaccine completion. In addition, prospective travelers may not fully understand the health implications of travel-related infections and the potential benefits of immunizations.

Some studies have shown that VFRs who seek pre-travel 
care are treated differently than non-VFRs. Among pediatric travelers in Boston, VFRs were less likely than non-VFRs to be prescribed atovaquone-proguanil and antidiarrheal medication. In addition, among those who indicated English as their primary language, non-VFRs were more likely to receive typhoid and yellow fever vaccine than VFRs. ${ }^{17}$ No significant differences were observed between VFRs and nonVFRs for antimalarial prescriptions, but a greater percentage of VFRs were prescribed antidiarrheal medications. This may be because more VFRs planned travel to Africa, Asia, and the Middle East, where antidiarrheal precautions are more strongly recommended. ${ }^{18}$

A limitation of the current study is the small number of VFRs; it was not possible to include VFRs who did not seek pre-travel care at the TTMC. It is possible that the VFRs included in this study were a relatively more motivated and health-literate subgroup; therefore, the differences in pretravel vaccinations between VFRs and non-VFRs may have been underestimated. Some travelers will not seek pre-travel consultation unless there is a requirement to enter a country, such as proof of yellow fever or meningococcal vaccination. This study has a lack data on whether prescriptions were filled and whether travelers adhered to antimalarial prophylaxis. This study was conducted at a single clinic, which limits the generalizability of the findings.

Nonetheless, the findings of this study highlight some disparities in pre-travel care for VFRs and highlight several opportunities to improve pre-travel education and vaccinations. Further research exploring specific reasons for incompletion of recommended vaccines will pinpoint changes to be made in practice and improve pre-travel health care. The potential impact of language barriers in adherence to travel recommendations also needs to be elucidated.

\section{Conclusion}

VFRs visiting the Mayo TTMC were more commonly younger travelers planning to visit African and Asian countries. They also had longer travel durations and lower rates of vaccine completion than non-VFRs. VFRs may be at higher risk of being affected by certain infectious diseases associated with travel. Travel clinics have the opportunity to provide focused pre-travel counseling and improved vaccinations for VFR travelers.

\section{Authors' Contributions}

EMT, JWN, and IGS participated in study design, protocol preparation, and manuscript writing. EMT and JRM performed data collection. DJJ, PMW, and CF performed data analysis and produced tables. DJS and MLW reviewed the manuscript.

\section{Conflict of Interest Disclosures}

The authors declare that they have no conflict of interest.

\section{Ethical Approval}

This study was approved by the Mayo Clinic Institutional Review Board. All patient data is presented in aggregate and with identifiers removed. This was a retrospective chart review and involved no patient contact or intervention.

\section{Research Highlights}

\section{What Is Already Known?}

VFRs form a distinct subgroup of international travelers with longer travel durations and lower rates of vaccine completion compared to non-VFRs.

\section{What This Study Adds?}

VFRs are less likely to complete rabies vaccination. The lower vaccination rates among VFRs illustrate disparities in pre-travel care. Improved pre-travel education could better elucidate the specific challenges that the VFR population faces.

\section{Funding/Support}

This study was funded by the Mayo Clinic Robert D. and Patricia E. Kern Center for the Science of Health Care Delivery and by the Division of Primary Care Internal Medicine, Mayo Clinic, Rochester, Minnesota.

\section{Acknowledgments}

This study was funded by the Mayo Clinic Robert D. and Patricia E. Kern Center for the Science of Health Care Delivery and the Division of Primary Care Internal Medicine, Mayo Clinic, Rochester.

\section{References}

1. Francyne Harrigan F, Seo SM. 244 million international migrants living abroad worldwide, new UN statistics reveal. Sustainable Development; 2016. http://www.un.org/sustainabledevelopment/ blog/2016/01/244-million-international-migrants-living-abroadworldwide-new-un-statistics-reveal/.

2. Dews F. What Percentage of the U.S. Population is Foreign Born? Vol 2017. Washington DC: Brookings; 2013.

3. Keystone JS. Immigrants Returning Home to Visit Friends \& Relatives (VFRs). In: Brunette GW, ed. CDC Health Information for International Travel 2014. New York: Oxford University Press; 2013.

4. Heywood AE, Zwar N, Forssman BL, et al. The contribution of travellers visiting friends and relatives to notified infectious diseases in Australia: state-based enhanced surveillance. Epidemiology and infection. 2016. doi:10.1017/S0950268816001734

5. Brower S. Demographic Overview of Minnesota. Paper presented at: Minnesota State Demographic Center; September 24, 2015, 2015; St. Paul, MN

6. Our Projections. Minnesota State Demographic Center website. St. Paul, MN; 2016. https://mn.gov/admin/demography/data-by-topic/ population-data/our-projections/.

7. Angell SY, Cetron MS. Health disparities among travelers visiting friends and relatives abroad. Ann Intern Med. 2005;142(1):67-72.

8. Van Herck K1, Van Damme P, Castelli F, et al. Knowledge, attitudes and practices in travel-related infectious diseases: the European airport survey. J Travel Med. 2004;11(1):3-8.

9. Bui YG, Trepanier S, Milord F, Blackburn M, Provost S, Gagnon S. Cases of malaria, hepatitis A, and typhoid fever among VFRs, Quebec (Canada). JTravel Med. 2011;18(6):373-378. doi:10.1111/ j.1708-8305.2011.00556.x.

10. Luise D, Dona D, Visentin F, Marini G, Giaquinto C, Cattelan A. Comparing imported malaria in adults and children presenting to an Italian teaching hospital: A 10-year retrospective study. Travel Med Infect Dis. 2017. doi:10.1016/j.tmaid.2017.04.008.

11. LaRocque RC, Deshpande BR, Rao SR, et al. Pre-travel health care of immigrants returning home to visit friends and relatives. Am J Trop Med Hyg. 2013;88(2):376-380. doi:10.4269/ 
ajtmh.2012.12-0460.

12. Pitzinger B, Steffen R, Tschopp A. Incidence and clinical features of traveler's diarrhea in infants and children. Pediatr Infect Dis J. 1991;10(10):719-723.

13. Chen LH, Wilson ME, Davis $\mathrm{X}$, et al. Illness in long-term travelers visiting GeoSentinel clinics. Emerg Infect Dis. 2009;15(11):17731782. doi:10.3201/eid1511.090945.

14. Monge-Maillo B, Norman FF, Perez-Molina JA, Navarro M, Diaz-Menendez M, Lopez-Velez R. Travelers visiting friends and relatives (VFR) and imported infectious disease: travelers, immigrants or both? A comparative analysis. Travel Med Infect Dis. 2014;12(1):88-94. doi:10.1016/j.tmaid.2013.07.004 .
15. Leder $\mathrm{K}$, Tong $\mathrm{S}$, Weld $\mathrm{L}$, et al. Illness in travelers visiting friends and relatives: a review of the GeoSentinel Surveillance Network. Clin Infect Dis. 2006;43(9):1185-1193. doi:10.1086/507893.

16. Gautret P, Parola P. Rabies vaccination for international travelers. Vaccine. 2012;30(2):126-133. doi:10.1016/j.vaccine.2011.11.007.

17. Han P, Yanni E, Jentes ES, et al. Health challenges of young travelers visiting friends and relatives compared with those traveling for other purposes. Pediatr Infect Dis J. 2012;31(9):915919. doi:10.1097/INF.0b013e318259efbe.

18. Diemert DJ. Prevention and self-treatment of traveler's diarrhea. Clin Microbiol Rev. 2006;19(3):583-594. doi:10.1128/ CMR.00052-05. 